\title{
OPTICAL SPECTROSCOPY ON THE SPIN-PEIERLS COMPOUND $\mathrm{CuGeO}_{3}$
}

\author{
PAUL H.M. VAN LOOSDRECHT \\ II Physikalisches Institut der RWTH-Aachen, Templergraben 55, D-52056 Aachen, Germany.
}

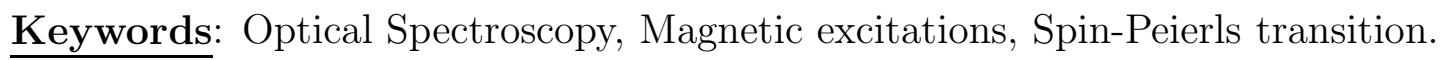

\begin{abstract}
An overview is given of Raman and infrared spectroscopic studies of the inorganic spin-Peierls compound $\mathrm{CuGeO}_{3}$, with an emphasis on the magnetic fluctuations in the uniform, dimerized, and high field phases of this quasi one dimensional magneto-elastic compound.
\end{abstract}

\section{Introduction}

One dimensional quantum spin systems show a variety of interesting physical phenomena such as low energy quantum fluctuations, pronounced soliton or spinon continua [1], and the occurrence of gapful states in Haldane [2], spin-Peierls [3], and ladder systems [4] systems. The magneto-elastic spin-Peierls (SP) transition in organic compounds has been studied widely in the 80's [5]. The discovery of an inorganic SP compound, $\mathrm{CuGeO}_{3}$, in 1993 [6] has led to a renewed interest in these compounds, which has been boosted once more by the discovery of a second inorganic $\mathrm{SP}$ compound $\mathrm{NaV}_{2} \mathrm{O}_{5}$ [7]. The inorganic nature of these compounds has opened the door to new investigations into the nature of SP compounds, which were difficult to perform in the past [8].

The SP transition is the magnetic analogon of the Peierls transition in one dimensional metallic systems [9]. It results from the instability of 1D antiferromagnetic Heisenberg spin chains at low temperatures towards dimerization due to the degeneracy of the ground state with the lowest spin excitations at $k_{d}=\pi[1]$. The coupling of the 1D magnetic chain to the 3D phonon system may lead to a phase transition (the SP transition [3]) which comprises a simultaneous dimerization of both the lattice and the magnetic system. In the presence of an external magnetic field, the spin system shows the tendency to build up a magnetization. As a consequence, the degeneracy of the spin excitations with the ground state tends to move away from $k_{d}=\pi$ [1], as will the wave vector of the lattice distortion. For low fields, however, the distortion is "pinned" at $k_{d}=\pi$ due to the presence of umklapp processes. At high enough fields the system will undergo a phase transition to a modulated phase by an abrupt jump of $k_{d}$ away from $\pi$. In $\mathrm{CuGeO}_{3}$ this has indeed been observed experimentally [10] In contrast to the dimerized phase, the lattice and spin systems now become modulated with a wave vector which is generally incommensurate with the periodicity of the undistorted or uniform phase.

Inelastic light scattering (ILS) from magnetic excitations in antiferromagnetic compounds has been known for a long time, and provides a sensitive tool to study properties such as magnetic excitation spectra, exchange interactions, symmetry, and phase transitions [11].

One of the most important inelastic light scattering mechanisms in antiferromagnetic compounds is the so called exchange interaction or two magnon scattering process [12, 13]. The Raman operator for such processes may be written as $R=\sum A_{i \vec{\delta}}\left(\vec{E}_{\text {in }} \cdot \vec{\delta}\right)\left(\vec{E}_{\text {out }} \cdot \vec{\delta}\right)$ [14], where $\vec{E}$ is the optical electric field vector, $i$ labels the magnetic spins, and $\vec{\delta}$ are the connecting vectors to the neighbors of spin $i$. The elements $A_{i \vec{\delta}}$ are symmetry dependent. For $A_{g}$ symmetries they 
are proportional to the exchange interaction between spin $i$ and spin $i+\delta$ in the direction of the polarization the optical electric field. In this case the Raman intensity will therefore roughly be proportional to the square of the exchange interaction, and in a one dimensional systems one expects magnetic scattering only when the incident and scattered light are both polarized along the chain direction.

Two magnon processes are also possible in infrared absorption experiments. So far, however, there have been no reports on the observation of this type of scattering in $\mathrm{CuGeO}_{3}$. It appears that in $\mathrm{CuGeO}_{3}$ the most important absorption processes are, like in EPR experiments, due to magnetic dipole transitions [15, 16].

In this contribution I will review some results of inelastic light scattering studies on $\mathrm{CuGeO}_{3}$ which have been reported over the past five years. The review is inevitably somewhat biased by our own work, although I do believe that all key papers have been included in this review.

\section{$2 \mathrm{CuGeO}_{3}$}

A convenient method to produce good quality single crystals of $\mathrm{CuGeO}_{3}$ is the traveling floating zone method 18. Using this technique one easily obtains large $\left(10 \times 5 \times 100 \mathrm{~mm}^{3}\right)$, high quality single crystals suitable for use in inelastic optical or neutron scattering experiments.

The room temperature structure of $\mathrm{CuGeO}_{3}$ is orthorhombic with space group $\mathrm{Pbmm}$, and unit cell parameters $a=4.81 \AA ; b=8.43 \AA ; c=2.95 \AA$ [19]. The structure is build of chains of planar $\mathrm{CuO}_{4}$ squares running along the $c$-direction, which are separated from each other by chains of $\mathrm{GeO}_{4}$ tetrahedra. The $k=0$ vibrational modes can be classified according to [20,21]:

$$
\begin{aligned}
\Gamma_{v i b}= & 4 A_{g} \oplus 4 B_{1 g} \oplus 3 B_{2 g} \oplus B_{3 g} \\
& 2 A_{u} \oplus 4 B_{1 u} \oplus 6 B_{2 u} \oplus 6 B_{3 u},
\end{aligned}
$$

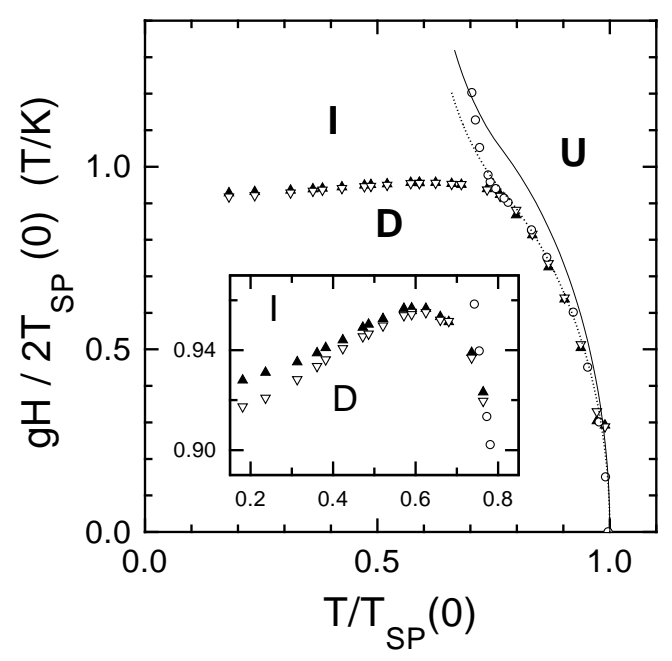

Figure 1: $\quad B-T$ Phase diagram of $\mathrm{CuGeO}$ taken from ref. [17].

where the gerade modes are Raman active and the ungerade $B$ modes are infrared active. For a more thorough discussion on the phonon scattering in $\mathrm{CuGeO}_{3}$ see for instance Popović et al. 21].

The magnetic ions in $\mathrm{CuGeO}_{3}$ are the $\mathrm{Cu}^{2+}$ ions (spin $S=1 / 2$ ) located on a fully symmetric site of the structure. The exchange interaction in the chain $(c)$ direction is determined by an almost $90^{\circ} \mathrm{Cu}-\mathrm{O}-\mathrm{Cu}$ superexchange path, leading to a relatively small effective nearest neighbor (nn) exchange integral of $J_{c} \sim 120 \mathrm{~K}$ [6,22]. Frustrating antiferromagnetic next nearest neighbor (nnn) interactions have been used to explain the observed deviations of the observed temperature dependence of the magnetic susceptibility $\mathrm{CuGeO}_{3}$ [23, 24], and may explain the observed temperature dependence of the magnetic susceptibility. The values reported for the frustration $\alpha=J_{c}^{n n n} / J_{c}^{n n}$ are quite high and range from 0.24 to 0.37 , with $J_{c}^{n n} \approx 160 \mathrm{~K} 23$ 25. It has been shown that under hydrostatic pressure the frustration may become even larger 25, 26.

The magnetic interactions between the magnetic chains is much smaller, though not negligible: $J_{b} \sim 0.1 J_{c} ; J_{a} \sim-0.01 J_{c}$. The presence of the interchain interaction leads to a sizable dispersion of the spin-excitations along the $b$-direction, and consequently to the occurrence of 
two magnetic gaps in the spin-Peierls phase [15, 16, 22, 27], situated at different points in the Brillouin zone [28] $\left(\Delta_{S P}=17 \mathrm{~cm}^{-1}\right.$ at $k=\left(k_{b}, K_{c}\right)=(0,0),(\pi, \pi)$, and $\Delta_{b}=44 \mathrm{~cm}^{-1}$ at $k=(0, p i),(\pi, 0))$.

The phase diagram of $\mathrm{CuGeO}_{3}$ has been determined by various authors [17, 29, 30]. It more or less follows the generic phase diagram predicted for a spin-Peierls compound [31, 32], although there are some serious deviations, in particular at high magnetic fields [30]. The phase diagram after [17] is reproduced in figure 1. It comprises a dimerized phase below $14 \mathrm{~K}$ and 12.5 $\mathrm{T}$, an incommensurate phase below $9 \mathrm{~K}$ and above $12 \mathrm{~T}$, and a uniform phase for higher temperatures. The uniform phase itself can be divided into two regimes [33]. At low temperatures $\left(T<T_{\max }\right.$, where $T_{\max } \approx 60 \mathrm{~K} \approx J_{c} / 2$ is the temperature where the magnetic susceptibility reaches its maximum.) there exist short range correlations in the spin system, whereas at high temperatures there are only weak correlations.

\section{The uniform phase}

The 12 Raman and 15 infrared active phonons of the high temperature phase have all been observed experimentally [20,21, 34]. In addition, the acoustic modes have been studied by Brillouin spectroscopy 35. Spin-dependent scattering in the HT-regime has been observed only in (cc)-polarized Raman scattering spectra [33, 36] as a strong quasi-elastic peak (see figure 2). This polarization selection rule is in excellent agreement with the one dimensional character of $\mathrm{CuGeO}_{3}$, and continues to hold in the dimerized and high field phases as well. The origin of the quasi elastic scattering is at present not very well understood. Initially it has been proposed [33 that this scattering may be due one dimensional diffusive behavior of the spin-excitations since there is no short or long range order at high temperature. Later it has also been suggested that the scattering is due to magnetic energy relaxation [36]. This latter type of scattering should have a distinct dependence on the momentum transfer in the

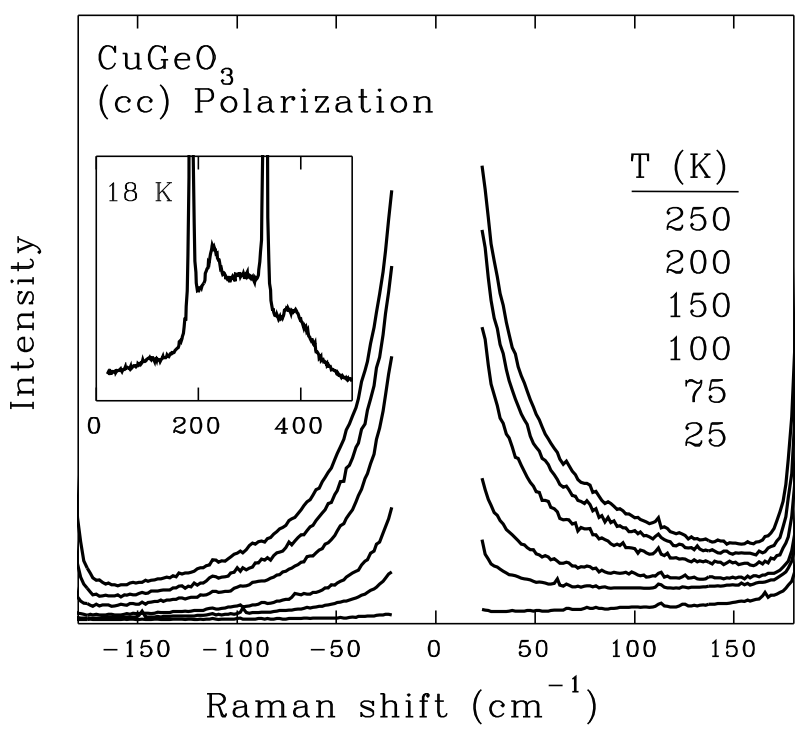

Figure 2: (cc)-Polarized Stokes and antiStokes Raman spectra of $\mathrm{CuGeO}_{3}$ in the uniform phase showing a broad, strongly temperature dependent quasi-elastic scattering. The inset shows the scattering continuum in the short range order regime of the uniform phase in (cc)polarization.

scattering process. Experimentally, however, the quasi-elastic scattering has no momentum transfer dependence at all [37]. Another possibility may be that the quasi-elastic scattering is due to spin-phonon processes, which have not yet been considered in literature.

Upon decreasing temperature (but still $T>T_{s p}$ ) the intensity of the QE peak decreases strongly. At low temperatures $\left(T_{s p}<T<J / 2\right)$ the QE peak has almost vanished, and one now observes only a broad band of excitations peaking around $230 \mathrm{~cm}^{-1}$ (see inset figure 2) [33, 38. The development of this continuum is due to the increasing short range spin-spin correlations in the (quasi-)one-dimensional spin system, which in turn leads to the formation of the spin-wave or spinon continuum [1]. The reason for the spin-scattering in the $A_{g}(c c)$ channel is not so clear. For a one dimensional nn Heisenberg chain one would not expect any scattering at all in this channel, since the exchange Raman operator commutes with the Hamiltonian 
itself. Muthukumar et al. 39 therefore proposed that the scattering is due to the presence of frustration in $\mathrm{CuGeO}_{3}$. Under this assumption they indeed found a remarkable agreement between their theory and the experimental results. Other origins of the scattering may be the two dimensionality of the system, or the presence of strong magneto-elastic interactions. Interestingly, it has been shown recently that taking spin-phonon interactions into account may lead to frustration in the magnetic system under the assumption that the typical phonon energies are much larger than the typical spin excitation energies [40].

\section{The dimerized phase}

Raman spectroscopic studies of the dimerized phase have been reported by several groups [33, 34, 38, 41]. The spin-Peierls transition leads to several new features in the (cc) polarized Raman spectra (see figure 3). In the first place, three zone boundary phonons appear in the (cc) spectra of the dimerized phase, at 105, 370, and $820 \mathrm{~cm}^{-1}$, which are activated either by magneto-elastic interactions or by the structural distortion [34, 42, 43]. Note that the $105 \mathrm{~cm}^{-1}$ mode shows a strong Fano lineshape distortion due to interactions with the spin excitations [33.

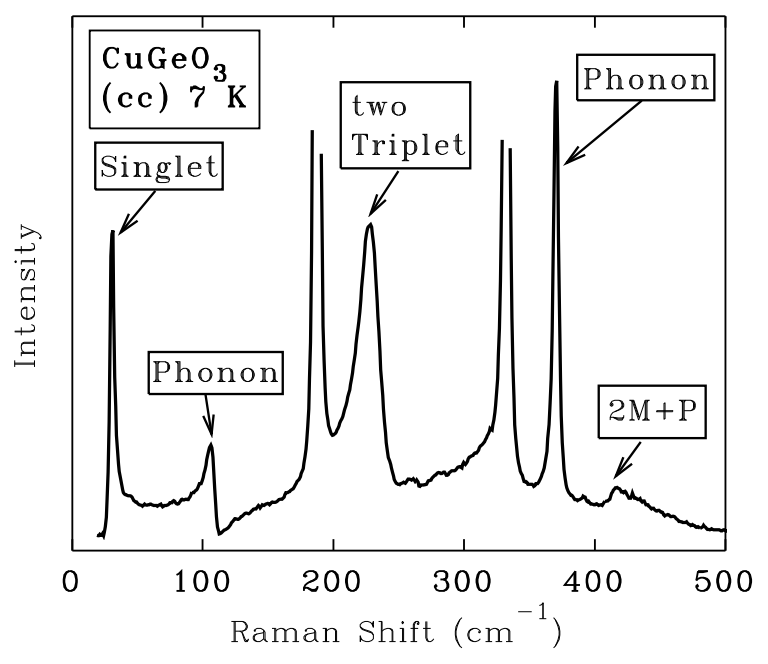

Figure 3: $\quad$ Part of the (cc)-Polarized Raman spectrum of $\mathrm{CuGeO}$ in the dimerized phase.
Secondly, a sharp, somewhat asymmetric mode appears in the Raman spectrum around $30 \mathrm{~cm}^{-1}$. This mode has been assigned to the $k=0$ singlet mode, which is a bound state of two antiparallel triplet excitations [44, 45]. The binding energy is of the order of $1-2 \mathrm{~cm}^{-1}$, and increases upon applying hydrostatic pressure 26].

Thirdly, a well defined continuum of scattered intensity can be observed in the spectra starting around $30 \mathrm{~cm}^{-1}$, and showing a sharp maximum at $228 \mathrm{~cm}^{-1}$. This scattering continuum is generally thought to be due to two magnon exchange scattering from the well defined triplet excitations in SP phase. Recent inelastic neutron scattering results, however, in the SP transition has an energy of about $228 \mathrm{~cm}^{-1}$ 46. Therefore, it seems likely that the peak at $228 \mathrm{~cm}^{-1}$ is due to mixed vibrational/magnetic excitations.

Finally there is a broad structure in the (cc) spectra above $230 \mathrm{~cm}^{-1}$, which extends up to $500 \mathrm{~cm}^{-1}$. This structure is most likely due to spin-phonon scattering processes. The peak at $417 \mathrm{~cm}^{-1}$ may, for instance, be assigned to a process where one $A_{g}\left(330 \mathrm{~cm}^{-1}\right)$ phonon, and two $k=(0, \pi)$ or $(\pi, 0)$ gap $\left(44 \mathrm{~cm}^{-1}\right)$ excitations are created.

It appears that one magnon scattering can not be observed for $\mathrm{CuGeO}_{3}$, probably because of the small spin-orbit coupling in $\mathrm{Cu}$. One magnon scattering would appear in the inelastic light scattering spectra as a peak around $17 \mathrm{~cm}^{-1}\left(=\Delta_{S P}\right)$. What has been observed at low energies using Brillouin spectroscopy is a thermally activated mode, arising from scattering between excited states [47], as well as an impurity induced mode in doped single crystals [41, 48].

The pressure dependent phase diagram of $\mathrm{CuGeO}_{3}$ has also been studied [26, 49 51]. At about $6 \mathrm{GPa}$ there is a structural phase transition to a monoclinic phase 49, above which no magnetic excitations have been observed in the Raman spectra 26, 51]. The SP transition temperature of $\mathrm{CuGeO}_{3}$ strongly increases upon applying hydrostatic pressure [52 54]. This 
has also been observed using Raman spectroscopy by monitoring the $370 \mathrm{~cm}^{-1}$ mode 51 or the singlet excitation [26]. The decrease of the peak energy of the two magnon continuum in the SP phase upon increasing pressure has led to the conclusion that the magnetic frustration in $\mathrm{CuGeO}_{3}$ has a large positive pressure coefficient, and that the SP phase transition is largely driven by frustration [26]. This is consistent with earlier thermodynamic experiments which led to the same conclusions 25].

There have been a few reports on infrared spectroscopy in the dimerized phase [16, 55, 56]. The main observations are a new absorption peak at $44 \mathrm{~cm}^{-1}$ [16,55], also seen in electron paramagnetic resonance spectroscopy [15], and the occurrence of new phonon modes [56] in the SP phase. Also the temperature dependence of the phonon spectrum has been studied [55, 56].

The $44 \mathrm{~cm}^{-1}$ absorption has been assigned to a magnetic dipole transition from the singlet ground state to the triplet gap mode. This interpretation, however, is problematic since the $k=0$ singlet-triplet gap is about $17 \mathrm{~cm}^{-1}$ [28, 57], and since normally singlet-triplet transitions are magnetic dipole forbidden. Nevertheless, the absorption behaves as a triplet in the sense that it indeed splits in a magnetic field (see figure 4) [16, 55, and its energy corresponds closely to the singlet-triplet gap at $k=(0, \pi)$ or $(\pi, 0)$ [22, 27]. The exact mechanism of the absorption process is at present unclear.

\section{The high field phase}

The high field, or incommensurate phase of $\mathrm{CuGeO}_{3}$ occurs for magnetic fields above $12.5 \mathrm{~T}$ and temperatures below about $9 \mathrm{~K}$ (see figure 1). There have been only a few reports on optical spectroscopy in the IC phase [16, 42, 55, 58.. In the dimerized phase, the presence of well defined triplet excitations led to a scattering continuum in the $A_{g}(c c)$ channel at energies between the singlet response at $30 \mathrm{~cm}^{-1}$, and the two magnon peak at 228 $\mathrm{cm}^{-1}$. In the IC phase, the singlet response disappears due to the closure (or decrease in energy ?) of the spin-Peierls gap. A clear two magnon peak is, though weaker, still observed [42, 58], indicating that there still exist more or less well defined, propagating triplet excitations in the center of the Brillouin zone with energies of the order of $\pi J$. Also the SP induced phonons observed in the $\mathrm{D}$ phase at 105 and $370 \mathrm{~cm}^{-1}$ persist in the IC phase, but again with a much weaker intensity. In fact,

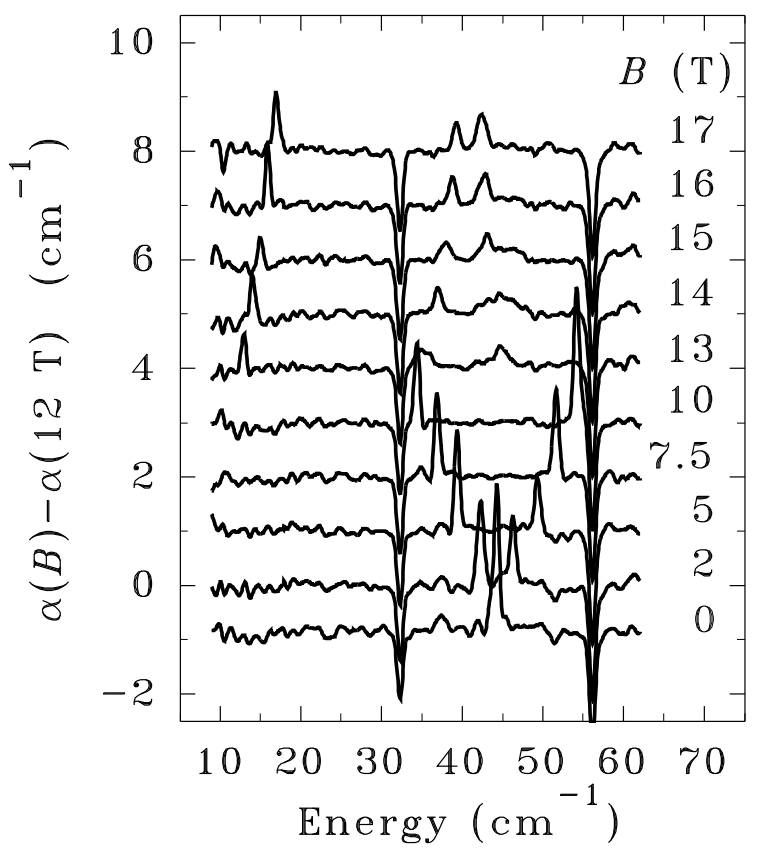

Figure 4: Absorbance difference spectra $\alpha(B)-$ $\alpha(12 \mathrm{~T})$ at $\mathrm{T}=2.3 \mathrm{~K}$ in the dimerized and incommensurate phases of $\mathrm{CuGeO}_{3}$. (ref. [10]) the temperature dependence of either the two-magnon or the induced phonons can be used to monitor the second order U-D or U-IC transitions, as well as the first order D-IC transition. At this latter transition, the intensities show, as expected for a first order transition, a step-like decrease upon entering the IC phase [58].

Like the disappearance of the singlet response in Raman spectroscopy, also the $44 \mathrm{~cm}^{-1}$ gap mode observed in infrared spectroscopy [16, 55] disappears in the IC phase. Instead, one now observes a sharp absorption with an low energy scaling linearly with the magnetic field (see 
figure 4 , mode at $13-17 \mathrm{~cm}^{-1}$ ). In contrast to the non-magnetic singlet ground state of the $\mathrm{D}$ phase, one expects a magnetic ground state in the IC phase. The low energy mode at 13 $\mathrm{cm}^{-1}$ (for $B=13 \mathrm{~T}$ ) has therefore been assigned to transitions between levels of the Zeeman splitted ground state. Far infrared spectra have also given direct evidence for a modulated structure in the IC phase by the appearance of absorption processes involving magnetic and vibrational excitations with $k=k_{i c}$ (where $k_{i c}$ is the modulation vector) in the $30-50 \mathrm{~cm}^{-1}$ region (see figure (1). The observation of an additional mode at $45 \mathrm{~cm}^{-1}$ for fields just above the D-IC phase transition strongly indicates the existence of a discommensurations regime in the IC phase for fields between 12.5 and $16 \mathrm{~T}$.

\section{Conclusions}

The overview given in this contribution shows that optical spectroscopies can give quite a lot of insight into the properties of low dimensional magnetic and/or magneto-elastic compounds. Many things have been discussed or touched upon such as spin and energy diffusion, spin excitation continua, spin-phonon processes, propagating triplet excitations, singlet bound states, magnetic frustration, phase transitions, and incommensurate structures. Nevertheless, some things had to be left out. The most important omissions are probably the temperature and pressure dependent optical studies the phonon spectra, and the investigations of the strong influence of that non-magnetic substitutions have on the properties of $\mathrm{CuGeO}_{3}$ [43, 48, 56, 59 62].

\section{Acknowledgments}

I thank Prof. J.-P. Boucher for introducing me to the subject of spin chain systems and to $\mathrm{CuGeO}_{3}$, and for many fruitful discussions. Sincere thanks are also due to the group of Prof. A. Revcolevschi and Prof. G. Dhalenne for growing excellent $\mathrm{CuGeO}_{3}$ single crystals, for making them available to us, and for stimulating discussions. Finally, thanks are due to all the people at the Grenoble High Magnetic Field Laboratory, the II Physikalisches Institut der RWTH-Aachen, and at the Institut für Theoretische Physik and II Physikalisches institut der Universität zu Köln for their numerous contributions and fruitful discussions.

\section{References}

[1] G. Müller, H. Thomas, H. Beck, and J. C. Bonner, Phys. Rev. B 24, 1429 (1981).

[2] F. D. M. Haldane, Phys. Rev. Lett. 50, 1153 (1983).

[3] E. Pytte, Phys. Rev. B 10, 4637 (1974).

[4] E. Dagotto and T. M. Rice, Science 271, 618 (1996).

[5] J. W. Bray, L. V. Iterrante, I. S. Jacobs, and J. C. Bonner, in Extended linear chain compounds, edited by J. S. Miller (Plenum Press, New York, 1983), Vol. 3.

[6] M. Hase, I. Terasaki, and K. Uchinokura, Phys. Rev. Lett. 70, 3651 (1993).

[7] M. Isobe and Y. Ueda, J. Phys. Soc. Jap. 65, 1178 (1996).

[8] J. P. Boucher and L. P. Regnault, J. Phys. I (paris) 6, 1939 (1996).

[9] R. E. Peierls, Quantum theory of solids (Oxford University Press, London, 1955).

[10] V. Kiryukhin and B. Keimer, Phys. Rev. B 52, R704 (1995).

[11] M. Cottam and D. Lockwood, Light Scattering in Magnetic Solids (John Wiley \& Sons, New York, 1986). 
[12] T. Moriya, J. Phys. Soc. Japan 23, 490 (1967).

[13] P. A. Fleury and R. Loudon, Phys. Rev. 166, 514 (1968).

[14] R. J. Elliot and M. F. Thorpe, J. Phys. C 2, 1630 (1969).

[15] T. M. Brill, J. P. Boucher, J. Voiron, G. Dhalenne, A. Revcolevschi, and J. P. Renard, Phys. Rev. Lett. 73, 1545 (1994).

[16] P. H. M. van Loosdrecht, S. Huant, G. Martinez, G. Dhalenne, and A. Revcolevschi, Phys. Rev. B 54, R3730 (1996).

[17] T. Lorenz, U. Ammerahl, T. Auweiler, B. Büchner, A. Revcolevschi, and G. Dhalenne, Phys. Rev. B 55, 5914 (1997).

[18] A. Revcolevschi and G. Dhalenne, Adv. Mater. 5, 9657 (1993).

[19] H. Völlenkle, A. Wittman, and N. Nowotny, Monatsh. Chem. 98, 1352 (1967).

[20] S. D. Dević, M. J. Konstantinović, Z. V. Popović, G. Dhalenne, and A. Revcolevschi, J. Phys.: Condens. Matter 6, L745 (1994).

[21] Z. Popović, S. D. Dević, V. N. Popov, G. Dhalenne, and A. Revcolevschi, Phys. Rev. B 52, 4185 (1995).

[22] M. Nishi, O. Fujita, and J. Akimitsu, Phys. Rev. B 50, 6508 (1994).

[23] G. Castilla, S. Chakravarty, and V. J. Emery, Phys. Rev. Lett. 75, 1823 (1995).

[24] J. Riera and A. Dobry, Phys. Rev. B 51, 16098 (1995).

[25] B. Büchner, U. Ammerahl, T. Lorenz, W. Brenig, G. Dhalenne, and A. Revcolevschi, Phys. Rev. Lett. 77, 1624 (1996).

[26] P. H. M. van Loosdrecht, J. Zeman, G. Martinez, G. Dhalenne, and A. Revcolevschi, Phys. Rev. Lett. 79, 487 (1997).

[27] L. P. Regnault, M. Aïn, B. Hennion, G. Dhalenne, and A. Revcolevschi, Phys. Rev. B 53, 5579 (1996).

[28] G. Uhrig, Phys. Rev. Lett. 79, 163 (1997).

[29] M. Hase, I. Terasaki, K. Uchinokura, M. Tokunaga, N. Miura, and H. Obara, Phys. Rev. B 48, 9616 (1993).

[30] T. Lorenz, B. Büchner, P. H. M. van Loosdrecht, A. Revcolevschi, and G. Dhalenne, (1998), unpublished.

[31] L. N. Bulaevskii, A. I. Buzdin, and D. I. Khomskii, Sol. St. Comm. 27, 5 (1978).

[32] M. C. Cross, Phys. Rev. B 20, 4606 (1979).

[33] P. H. M. van Loosdrecht, J. P. Boucher, G. Martinez, G. Dhalenne, and A. Revcolevschi, Phys. Rev. Lett. 76, 311 (1996).

[34] H. Kuroe, T. Sekine, M. Hase, Y. Sasago, K. Uchinokura, H. Kojima, I. Tanaka, and Y. Shibuya, Phys. Rev. B 50, 16468 (1994).

[35] H. Yamaguchi, M. Tamaguchi, and T. Yagi, J. Phys. Soc. Jpn. 64, 1055 (1995).

[36] H. Kuroe, J. i. Sasaki, T. Sekine, N. Koide, Y. Sasago, K. Uchinokura, and M. Hase, Phys. Rev. B 55, 409 (1997).

[37] M. Fischer et al., unpublished (1997).

[38] P. Lemmens, B. Eisener, M. Brinkmann, L. V. Gasparov, G. Güntherodt, P. v. Dongen, M. Weiden, W. Richter, C. Geibel, and F. Steglich, Physica B 223-224, 535 (1996). 
[39] V. N. Muthukumar, C. Gros, W. Wenzel, R. Valenti, P. Lemmens, B. Eisener, G. Güntherodt, M. Weiden, C. Geibel, and F. Steglich, Phas. Rev. B 54, R9635 (1996).

[40] G. S. Uhrig, unpublished (1997).

[41] I. Loa, S. Gronemeyer, C. Thomsen, and R. K. Kremer, Sol. St. Comm. 99, 231 (1996).

[42] P. H. M. van Loosdrecht, J. P. Boucher, S. Huant, G. Martinez, G. Dhalenne, and A. Revcolevschi, Physica B 230-232, 1017 (1997).

[43] M. Fischer, P. H. M. van Loosdrecht, P. Lemmens, G. Güntherodt, B. Büchner, T. Lorenz, M. Breuer, J. Zeman, G. Martinez, G. Dhalenne, and A. Revcolevschi, unpublished (1997).

[44] G. S. Uhrig and H. J. Schulz, Phys. Rev. B 54, R9624 (1996).

[45] G. Bouzerar, A. P. Kampf, and F. Schönfeld, cond-mat/9701176 (1997).

[46] M. Braden, Private communication (1997).

[47] G. Els, P. H. M. van Loosdrecht, P. Lemmens, H. Vonberg, G. Güntherodt, G. S. Uhrig, O. Fujita, J. Akimitsu, G. Dhalenne, and A. Revcolevschi, Phys. Rev. Lett. (in press) (1997).

[48] G. Els et al., unpublished (1997).

[49] D. M. Adams, J. Haines, and S. Leonard, J.Phys.: Condens. Matter 3, 5183 (1991).

[50] A. Jayaraman, S. Y. Wang, L. C. Ming, and S. W. Cheong, Phys. Rev. Lett. 75, 2356 (1995).

[51] A. R. Goñi, T. Zhou, U. Schwarz, R. K. Kremer, and K. Syassen, Phys. Rev. Lett. 77, 1079 (1996).

[52] H. Winkelman, E. Gamper, B. Büchner, M. Braden, A. Revcolevschi, and G. Dhalenne, Phys. Rev. B 51, 12884 (1995).

[53] H. Takahashi, N. Mori, O. Fujita, J. Akimitsu, and T. Matsumoto, Sol. St. Comm. 95, 817 (1995).

[54] M. Nishi, O. Fujita, J. Akimitsu, K. Kakurai, and Y. Fujii, Phys. Rev. B 52, R6959 (1995).

[55] G. Li, J. L. Musfeldt, Y. J. Wang, S. Jandl, M. Poirier, A. Revcolevschi, and G. Dhalenne, Phy. Rev. B 54, R15633 (1996).

[56] A. Damascelli, D. van der Marel, F. Parmigiani, G. Dhalenne, and A. Revcolevschi, condmat/9708026 (1997).

[57] M. Nishi, O. Fujita, and J. Akimitsu, Physica B 210, 149 (1995).

[58] P. H. M. van Loosdrecht, J. P. Boucher, G. Martinez, G. Dhalenne, and A. Revcolevschi, J. Appl. Phys. 79, (1996).

[59] P. Lemmens, M. Fischer, G. Güntherodt, C. Gros, P. G. J. van Dongen, M. Weiden, W. Richter, C. Geibel, and F. Steglich, Phys. Rev. B 55, 15076 (1997).

[60] J. J. McGuire, T. Rõõm, T. E. Mason, T. Timusk, H. Dabkowska, S. M. Coad, and D. M. Paul, cond-mat/9707163 (1997).

[61] S. D. Dević, Z. V. Popović, Y. Raptis, G. Dhalenne, and A. Revcolevschi, This volume (1997).

[62] A. Damascelli, D. van der Marel, F. Parmigiani, G. Dhalenne, and A. Revcolevschi, Physica B (in press) (1997). 